\title{
FAILURE BEHAVIOR OF SAND UNDER PRINCIPAL STRESS AXES ROTATION CONDITION
}

\author{
Jian dong Wang ${ }^{1, a}$, Xue feng $\mathrm{Li}^{1, b}$, Yu qi He ${ }^{1, c}$ \\ ${ }^{1}$ Solid Mechanics Institute, Ningxia University, Yinchuan 750021, China. \\ a861441175@qq.com, blixuefeng1928@163.com, c1029571443@qq.com
}

\begin{abstract}
Keywords: sand, micro fabric, macro-micro incorporation, principal stress axes rotation, anisotropy strength.

Abstract. In order to describe the failure behavior of sand involving the rotation of principal stress axes, a failure criterion with the method of macro-micro incorporation is employ in this paper. Considering the geometric relationship of loading stress and sand micro fabric, the proposed failure criterion can describe the effect of microstructure on failure properties under any angle rotation of principal stress condition. The verification with hollow cylindrical test results show that established expression can describe the failure behavior with different angle of loading stress directions. The different failure behavior caused by sand anisotropy under the principal stress axes rotation conditions is verified preliminarily.
\end{abstract}

\section{Introduction}

Rotation of principal stress axes is a common aspect of many loading situations in geotechnical engineering, such as those caused by earthquakes, vehicular traffic and sea waves loading. A different mechanical properties show between the rotation and fixed direction of principal stress, for example, which will cause significant changes of the strength and deformation. Showing different strength behaviour in different directions, the fundamental reason is anisotropy of the sand. In essence, the mechanical properties under the principal stress axes rotation belong to the part of anisotropy study for sand.

The tests showed that underlying mechanism of sand anisotropy can be attributed to its microscopic structural properties. Investigations at the microscopic level, a random packing of nonspherical particles, such as is found in sand, possesses statistical characteristics in the spatial arrangement of the particles and associated voids. These properties are collectively termed the material fabric [3, 2, 12]. Oda and Nakayama [6] listed three sources of inherent anisotropy in a granular material: (1) anisotropic distribution of contact normals, which is indicative of the relationships between particles; (2) the preferred orientation of void spaces; and (3) the preferred orientation of nonspherical particles. The material fabric describes inherent anisotropy of sand by a fabric tensor of second order [8]. The anisotropic parallel alignment of particles is universally observed not only in river, beach and coastal dune sands but also in artificially deposited sand [7]. Based on that sand micro feature is the internal mechanism of macroscopic phenomena, many scholars have launched a lot of tests with microscopic features, such as test $[10,13]$ show that sand micro fabric controls significantly the strength characteristics of macroscopic anisotropy, in particular, when the principal stress axes rotation, the strength change is very significant. Therefore, micro fabric is introduced to establish anisotropic failure criterion [15, 11], and gradually the method of macro-micro incorporation is formed to describe the sand anisotropy.

Anisotropy of sand will inevitably lead to different patterns of failure under principal stress axes rotation condition. However, the mechanical properties under principal stress axes rotation condition are mostly concentrated in the deformation law [1], the description of strength law is very rare. Presented here is the method of macro-micro incorporation to capture the failure behavior of principal stress direction rotation. A novel anisotropy state variable is properly defined and employed to establish failure expression. Further verifications are made via a series of hollow cylindrical test results. 


\section{The description under principal stress axes rotation condition}

\section{Anisotropic failure criterion of sand}

The failure criterion [14] with the method of macro-micro incorporation is employed in this paper as

$$
F=q-\exp (\zeta A) M_{f} g\left(\theta_{\sigma}\right) p=0 .
$$

Where $p$ is mean effective stress; $\zeta$ a weighting coefficient, usually the value of $0.2, q$ is deviator stress, $g\left(\theta_{\sigma}\right)$ is a function of $\theta_{\sigma}, \theta_{\sigma}$ is lode angle, $M_{f}$ is the stress ratio at peak failure , $M_{f}=6 \sin \phi_{f} /\left(3-\sin \phi_{f}\right)$ with $\phi_{f}$ being the peak friction angle derived from conventional traxial compression tests. The following definition of $g\left(\theta_{\sigma}\right)$ adopt the expression proposed by William and Warnkle [4]. $A$ is an novel anisotropic state variable proposed by $\mathrm{Li}$ et al[8], if the material is isotropic, then $A=0$, isotropic failure criterion can be described, if the material is anisotropic, $A$ is function of stress state, the degree of sand anisotropy and the orientation of the soil fabric relative to the loading direction, so equation(1) can describe that sand micro-features affect on failure criterion. There is the detailed description of failure criteria in the literature [14].

\section{Conversion relationship}

When the principal stress axes relative sand bodies rotate, and/or the reference frame has changed, the components of the fabric tensor will be subjected to the loading direction, as follows

$$
F_{i j}=C_{k i} C_{l j} F_{k l}^{\prime} .
$$

Where $C_{i j}$ is the cosine of the angle between the loading direction and fabric direction, $F_{i j}^{\prime}$ is the fabric tensor. In most practical cases, soils are transversely isotropic, only one scalar quantity is needed to define the fabric tensor. Oda and Nakayama [6] showed that the fabric tensor for such a material can be written in the following form:

$$
F_{i j}^{\prime}=\frac{1}{3+a}\left(\begin{array}{ccc}
1-a & 0 & 0 \\
0 & 1+a & 0 \\
0 & 0 & 1+a
\end{array}\right) .
$$

Where $a$ is a measurable quantity, called the vector magnitude [2], which indicates the magnitude of the anisotropy of the preferred orientation of the particles. It is clear that $a$ depends on particle shape and the process of soil deposition. Eq. (3) shows that $a$ varies from zero, when the material is isotropic, to unity, when the major axes of all the particles are uniformly distributed in the one plane.

In an orthogonal system all the angles are right angles, in Eq. (2), this places restrictions on the direction cosine matrix.

$$
(C)=\left(\begin{array}{lll}
C_{11} & C_{12} & C_{13} \\
C_{21} & C_{22} & C_{23} \\
C_{31} & C_{32} & C_{33}
\end{array}\right) .
$$

There will be nine equations of the following form.

$$
C_{i k} C_{j k}=C_{k i} C_{k j}=\delta_{i j} .
$$

Where $i, j, k=1,2,3$, and is the Kronecker. $\delta_{i j}=1$ if $i=j$, and $\delta_{i j}=0$ if $i \neq j$.

\section{Experimental validation}

In the Fig.2, the tests[5] were performed by increasing the shear stress $q$ monotonically until failure along fixed principal stress directions of $\beta=15^{\circ}, 30^{\circ}, 45^{\circ}, 60^{\circ}$ and $70^{\circ}, p=98 \mathrm{kPa}$, 
intermediate principal stress coefficient $b=0.5$. Test results and stress path shown in Fig.2. As show in Fig.2, the failure surface close to a circle, but the center of circle is not the origin point, mainly due to the presence of anisotropy.

Experiments show that the points of pure rotation of principal stress and combined loading condition (involving simultaneous increase in shear stress level and rotation of principal stress direction) very near the failure surface for the monotonic tests. Therefore, Description of failure surface under the rotation of principal stress direction condition is essential.

From the observation of test results, the failure criterion proposed by Gutierrez et al [5].

$$
F=\sqrt{\left(X-c_{x} p\right)^{2}+Y^{2}}-r_{f} p=0 .
$$

The failure surface defined above is a circle in the $\mathrm{X}-\mathrm{Y}$ stress space with its center located at $c_{x} p$ and with a radius $r_{f} p$. For isotropic sand, and $r_{f}=\sin \phi_{f}$. The parameter $c_{x}$ serves as a measure of the initial anisotropy of sand. A comparison of Eq. (15) and the measured failure points is shown in Fig. 2 which indicates a satisfactory representation of the test data. Although the proposed failure criterion can better describe the experimental results, but because of his establishment on the basis of test data summary, there are still some shortcomings, such as no describing microscopic properties, less clear physical meaning and different forms of failure criteria between principal stress rotation and fixed conditions. Therefore it is necessary to use the anisotropic failure criterion with the method of macro-micro incorporation to describe.

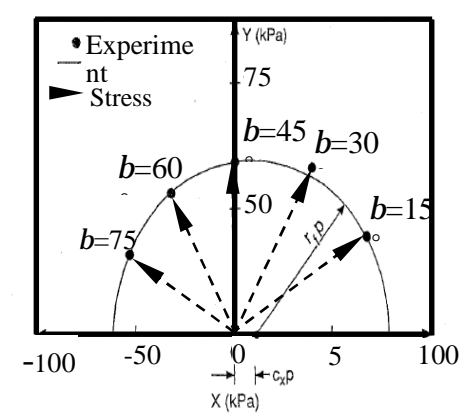

Fig.1 Experimental failure point and model failure surface

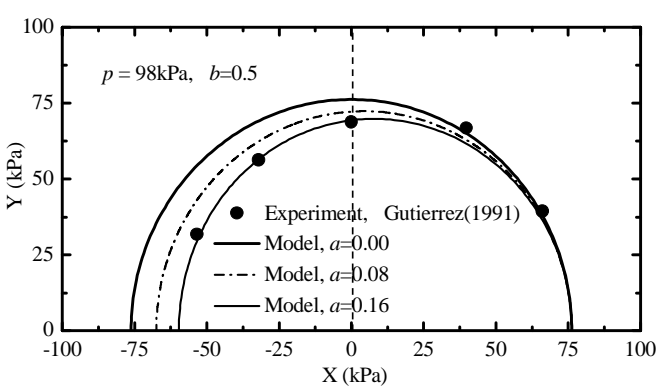

(b)

Fig. 2 is comparison of anisotropic failure criterion Eq. (13) and test results. According to the test of Gutierrez et al [5], $\phi_{f}=45^{\circ}$ and $c=0.73$, lacking of microscopic observations, refer to quantitative observations of micro-structure for Toyoura sand [16], take $a=0.00,0.08$, and 0.16 to simulate. As seen in Fig.2, when $a=0.00$, the failure curve for the origin of the circle is isotropic, when $a=0.08$, the failure surface started to deviate from the origin, the greater deviation, the greater values, when $a=0.16$, it close to the experimental values.

\section{Conclusions}

Because the microscopic anisotropy causes the different failure behavior under the principal stress axes rotation conditions, the failure criterion with the method of macro-micro incorporation employed to analyze the behavior. Based on the proposed failure criterion, the express of principal stress direction rotation is established, which is the function of stress state, the degree of sand anisotropy and the orientation of the soil fabric relative to the loading direction. When the material is anisotropic, failure behavior of the rotation of principal stress axes can be described by proposed expression with the microscopic characteristics of sand, and the smaller anisotropy, the smaller effect in this conditions. When the material is isotropic, the principal stress axes of rotation will not cause any changes of failure strength. Experimental validation show that proposed expression can better describe the failure under principal stress axes rotation conditions. 


\section{Acknowledgements}

This work was financially supported by Program of National Natural Science Funds (NSFC through grant No. 50979037), These supports are gratefully acknowledged.

\section{References}

[1] J. G. Qian, M. S. Huang. "Non-coaxial plastic flow theory in multi-dimensional stress state". Chinese Journal of Rock Mechanics and Engineering, 25(6), pp. 1259-1264, (2006).

[2] J. R. Curray. "Analysis of two-dimensional orientation data." The Journal of Geology, 64, pp. 117-131, (1956).

[3] J. R. F. Arthur and A. B. Phillips. "Correspondence: inherent anisotropy in sand". Geotechnique, 22(3), pp. 537-539, (1972).

[4] K. J. William, E. P. Warnke. "Constitutive model for the triaxial behavior of concrete". Proceedings of International Association for Bridge and Structure Engineering. Bergamo, Italy, 19, pp. 117-131, (1975).

[5] M. Gutierrez, K. Ishihara, I. Towhata. "Flow theory for sand during rotation of principal stress direction”. Soils and Foundations, 31(4), pp. 121-132, (1991).

[6] M. Oda, H. Nakayama. "Introduction of inherent anisotropy of soils in the yield function." Micromechanics of granular materials, M. Satake and J. T. Jenkins, eds., Elsevier, Amsterdam, pp. 81-90, (1988).

[7] M. Oda, I. Koishikawa, T. Higuchi. "Experimental study on anisotropic shear strength of sand by plane strain test". Soils and Foundations, 18(1), pp. 25-38, (1978).

[8] M. Oda. "Fabric tensor and its geometrical meaning." Introduction to mechanics of granular materials, M. Oda and K. Iwashita,ds., A.A. Balkema, Rotterdam, The Netherlands, pp. 27-35, (1999).

[9] M. Oda. "Initial fabrics and their relations to mechanical properties of granular materials." Soils and Foundations, 12(1), pp. 17-36, (1972).

[10]P. J. Guo. "Modified direct shear test for anisotropic strength of sand". Journal of Geotechnical and Environmental engineering. ASCE, 134 (9), pp. 1311-1318, (2008).

[11]P. V. Lade. "Failure criterion for cross-anisotropic soils". Journal of Geotechnical and Geoenvironmental Engineering, ASCE, 134(1), pp. 117-124, (2008).

[12]R. Brewer. "Fabric and mineral analysis of soils", Wiley, New York, (1964).

[13]T. B. S. Pradhan, F. Tatsuoka, N. Horii. "Strength and deformation characteristics of sand in torsional simple shear". Soils and Foundations, 28(3), pp.131-148, (1985).

[14]X. F. Li, M. S. Huang, J. G. Qian. "Failure criterion of anisotropic sand with the method of macro-micro incorporation". Chinese Journal of Rock Mechanics and Engineering, 29(9), pp. 18851892, (2010).

[15]Z. W. Gao, J. D. Zhao, Y. P. Yao. "A generalized anisotropic failure criterion for geomaterials". International Journal of solids and structures, 47(22-23), pp. 3166-3185, (2010).

[16]Z. X. Yang, X. S. Li, J. Yang. "Quantifying and modelling fabric anisotropy of granular soils". Geotechnique, 58(4), pp. 237-248, (2008). 